\title{
STRESS DISTRIBUTION AT THE RIGID CIRCULAR ARC INCLUSION END
}

\author{
LIU YOU-WEN \\ Department of Applied Mathematics and Mechanics, Central South University of Technology, \\ Changsha 410083, P.R.C. \\ JIANG CHI-PING \\ Division 12, Institute of Mechanics, Chinese Academy of Sciences, Beijing 100080, P.R.C.

\begin{abstract}
The elastic plane problem of a rigid co-circular arc inclusion under arbitrary loads is dealt with. Applying Schwarz's reflection principle integrated with the analysis of the singularity of complex stress functions, the general solution of the problem is found and several closed-form solutions to some problems of practical importance are given. Finally, the stress distribution at the arc inclusion end is examined and a comparison is made with that of the rigid line inclusion end to show the effect of curvature.
\end{abstract}

\section{INTRODUCTION}

THE PRESENCE of inclusions plays an important role in the fracture behavior of engineering materials, especially composites. In analyzing such problems, certain idealizations concerning the geometry and the mechanical properties of the constituent materials are usually made in order to be tractable mathematically. For an elastic plane problem, if the value of the elastic modulus of a flat inclusion is much greater than that of the matrix, it appears reasonable to consider it as a rigid line. Furthermore, from the viewpoint of inhomogeneities in solids, a rigid line and a slit crack are the two extreme cases of a flat inhomogeneity, i.e. for a rigid line $E \rightarrow \infty$ and for a crack $E \rightarrow 0$, where $E$ is the Young's modulus. It has been shown that rigid line inclusions also have singular fields at the tip (with the same square root singularity as the crack). Since Eshelby's paper [1], some investigations on the rigid straight line inhomogeneity problem have been made. In ref. [2], the solution for an elastic plane containing a rigid line under an arbitrary uniform stress state at infinity can be found. Recently, Hao and Wu [3] and Jiang [4] studied the elastic plane problem of collinear rigid lines, and Markenscoff and Dundurs [5] found weight functions for rigid line inclusions. However, to our knowledge, the rigid curve inclusion problem has not been solved as yet.

In the following an attempt is made to find the solution for an elastic plane with rigid co-circular arc inclusions under most general loading conditions, which include both an arbitrary uniform stress state at infinity and a concentrated load at an arbitrary point. Applying Schwarz's reflection principle integrated with the analysis of the singularity of complex stress functions [6], the general solution of the problem is found and several closed-form solutions to some problems, which may have some practical importance, are given.

In particular, the solution for a concentrated load at an arbitrary point is obtained. In addition to providing solutions to such practical problems as rivet loading or wedge loading at an arbitrary angle, this solution can be used as Green's function to obtain the stresses in the elastic plane with any given distribution of loads.

Finally, the stress distribution at the arc inclusion end is examined and a comparison is made with that of the rigid line inclusion end to show the effect of curvature.

\section{DESCRIPTION OF THE PROBLEM}

The problem to be considered is illustrated in Fig. 1. In an infinite elastic plane, a series of rigid arcs (i.e. rigid circular arc inclusions) are placed along a part, $L$, of a circle with radius $R$, where $L$ is a union of arcs $L_{j}$ with the end points $a_{j}$ and $b_{j}(j=1, \ldots, n) ; L^{\prime}$ is the remainder of the circle (Fig. 1). A concentrated force $P+i Q$ and a moment $m$ are applied at an arbitrary point $z_{0} . \sigma_{1}$ and $\sigma_{2}$ are the principal stresses at infinity and $\alpha$ is the angle between $\sigma_{1}$ and the real axis. 


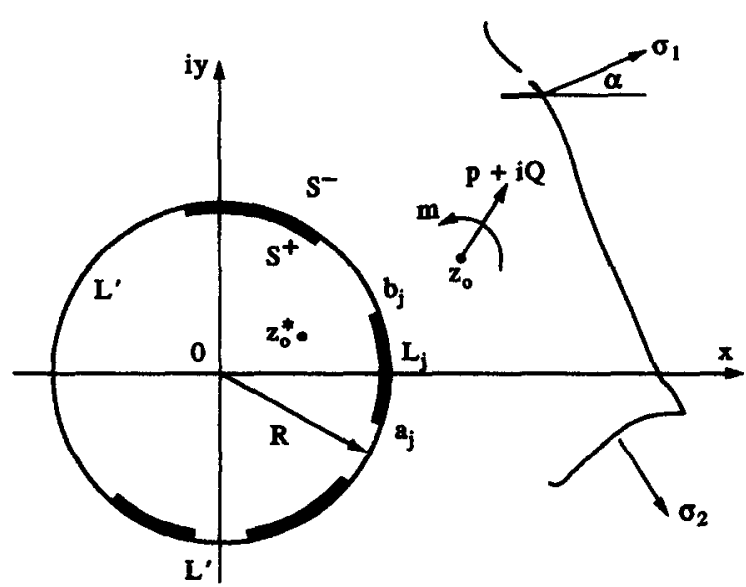

Fig. 1. Rigid co-circular arc inclusion in an elastic plane.

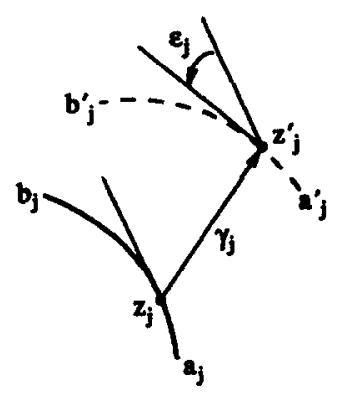

Fig. 2. Displacement and rotation of the rigid arc inclusion.

Let $u$ and $v$ be displacement components in Cartesian coordinates, then the boundary conditions for the problem may be expressed as follows:

$$
(u+i v)^{+}=(u+i v)^{-}= \begin{cases}\gamma_{1}+i \epsilon_{1}\left(z-z_{1}\right) & \text { on } L_{1} \\ \cdots \cdots & \text { on } L_{n}, \\ \gamma_{n}+i \epsilon_{n}\left(z-z_{n}\right)\end{cases}
$$

where the superscripts + and - refer to the values of the functions on the circle as approached from its inside $\left(S^{+}\right)$and outside $\left(S^{-}\right)$, respectively. The complex constants $\gamma_{j}$ denote the small translations of rigid arcs $L_{j}$ at certain points $z_{j}$, and real constants $\epsilon_{j}$ the small rotations of $L_{j}$ (Fig. 2).

Additionally, the equilibrium conditions of rigid arcs must be considered in order to determine solely the solution to the problem. Assuming that all rigid arcs are traction-free, then every resultant vector of the internal forces, with which every rigid arc acts on the matrix, vanishes and so does every resultant moment of these internal forces. Let $\sigma_{r}, \sigma_{\theta}$ and $\tau_{r \theta}$ be stress components in polar coordinates $(r, \theta)$; from Fig. 3, it is seen that

$$
\begin{gathered}
\int_{a_{j}}^{b_{j}}\left[\left(\sigma_{r} \cos \theta-\tau_{r \theta} \sin \theta\right)+i\left(\sigma_{r} \sin \theta+\tau_{r \theta} \cos \theta\right)\right]^{+} \mathrm{d} s-\int_{a_{j}}^{b_{j}}\left[\left(\sigma_{r} \cos \theta-\tau_{r \theta} \sin \theta\right)\right. \\
\left.+i\left(\sigma_{r} \sin \theta+\tau_{r \theta} \cos \theta\right)\right]^{-} \mathrm{d} s=0 \quad j=1, \ldots, n \\
\int_{a_{j}}^{b_{j}} R \tau_{r \theta}^{+} \mathrm{d} s-\int_{a_{j}}^{b_{j}} R \tau_{r \theta}^{-} \mathrm{d} s=0 \quad j=1, \ldots, n .
\end{gathered}
$$

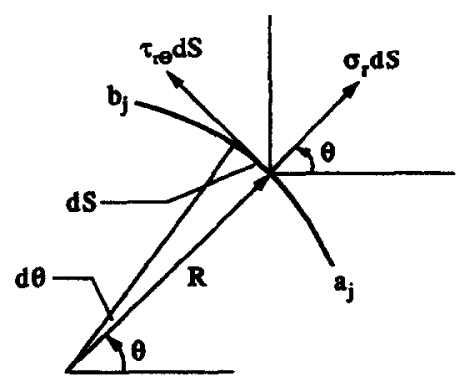

Fig. 3. Internal forces between the rigid arc inclusion and the matrix. 
Let $t=R \mathrm{e}^{i \theta}$ be the coordinate on the circle, then $\mathrm{d} s=R / i t \mathrm{~d} t$, and (2) and (3) can be written as:

$$
\begin{gathered}
\int_{a_{j}}^{b_{j}}\left(\sigma_{r}+i \tau_{r \theta}\right)^{+} \mathrm{d} t-\int_{a_{j}}^{b_{j}}\left(\sigma_{r}+i \tau_{r \theta}\right)^{-} \mathrm{d} t=0 \quad j=1, \ldots, n \\
R_{\mathrm{e}} \int_{a_{j}}^{b_{j}}\left(\sigma_{r}+i \tau_{r \theta}\right)^{+} \frac{\mathrm{d} t}{t}-R_{\mathrm{e}} \int_{a_{j}}^{b_{j}}\left(\sigma_{r}+i \tau_{r \theta}\right)^{-} \frac{\mathrm{d} t}{t}=0 \quad j=1, \ldots, n .
\end{gathered}
$$

\section{GENERAL SOLUTION}

To formulate the problem, we use the complex stress functions $\Phi(z)$ and $\Psi(z)$, in terms of which the stress components $\left(\sigma_{r}, \sigma_{\theta}, \tau_{r \theta}\right)$ in polar coordinates and the displacement components $(u, v)$ in Cartesian coordinates are given as [2]

$$
\begin{gathered}
\sigma_{r}+\sigma_{\theta}=2[\Phi(z)+\overline{\Phi(z)}] \\
\sigma_{r}+i \tau_{r \theta}=\Phi(z)+\overline{\Phi(z)}-\bar{z} \overline{\Phi^{\prime}(z)}-\frac{\bar{z}}{z} \overline{\Psi(z)} \\
2 \mu\left(u^{\prime}+i v^{\prime}\right)=i z\left[\kappa \Phi(z)-\overline{\Phi(z)}+\bar{z} \overline{\Phi^{\prime}(z)}+\frac{\bar{z}}{\bar{\Psi}} \overline{\Psi(z)}\right],
\end{gathered}
$$

where

$$
u^{\prime}=\frac{\partial u}{\partial \theta} \quad v^{\prime}=\frac{\partial v}{\partial \theta}
$$

$\mu$ is the shear modulus and $\kappa=3-4 v$ for plane strain, $\kappa=(3-v) /(1+v)$ for generalized plane stress, $v$ being Poisson's ratio. $\Phi(z)$ and $\Psi(z)$ are holomorphic in the entire plane cut along $L$, except at concentrated load points which are poles of the functions. For the problem under consideration we have [2]

$$
\begin{gathered}
\Phi(z)=\frac{M}{z-z_{0}}+\Gamma+\Phi_{0}(z) \\
\Psi(z)=\frac{N}{z-z_{0}}+\frac{\bar{z}_{0} M+M_{0}}{z-z_{0}}+\Gamma^{\prime}+\Psi_{0}(z),
\end{gathered}
$$

where $\Phi_{0}(z)$ and $\Psi_{0}(z)$ are holomorphic in the entire plane cut along $L$ and they vanish at infinity, and

$$
\begin{gathered}
M=-\frac{P+i Q}{2 \pi(1+\kappa)}, \quad N=\frac{\kappa(P-i Q)}{2 \pi(1+\kappa)}, \quad M_{0}=-\frac{i m}{2 \pi} \\
\Gamma=\bar{\Gamma}=\frac{1}{4}\left(\sigma_{1}+\sigma_{2}\right), \quad \Gamma^{\prime}=-\frac{1}{2}\left(\sigma_{1}-\sigma_{2}\right) \mathrm{e}^{-2 i x},
\end{gathered}
$$

where the rotation at infinity is assumed to vanish.

By applying Schwarz's reflection principle, a new analytical function $\Omega(z)$ can be defined [6]:

$$
\Omega(z)=\bar{\Phi}\left(\frac{R^{2}}{z}\right)-\frac{R^{2}}{z} \bar{\Phi}^{\prime}\left(\frac{R^{2}}{z}\right)-\frac{R^{2}}{z^{2}} \bar{\Psi}\left(\frac{R^{2}}{z}\right),
$$

where $\bar{\Phi}\left(R^{2} / z\right)=\overline{\Phi\left(R^{2} / \bar{z}\right)}$. Thus, (7) and (8) can be rewritten as

$$
\begin{gathered}
\sigma_{r}+i \tau_{r \theta}=\Phi(z)+\Omega\left(\frac{R^{2}}{\bar{z}}\right)+\bar{z}\left(\frac{\bar{z}}{R^{2}}-\frac{1}{z}\right) \overline{\Psi(z)} \\
2 \mu\left(u^{\prime}+i v^{\prime}\right)=i z\left[\kappa \Phi(z)-\Omega\left(\frac{R^{2}}{\bar{z}}\right)-\bar{z}\left(\frac{\bar{z}}{R^{2}}-\frac{1}{z}\right) \overline{\Psi(z)}\right] .
\end{gathered}
$$


From (10), (11) and (14), it is seen that

$$
\Omega(z)=-\frac{\bar{N}}{z}+\frac{\bar{N}}{z-z_{0}^{*}}-\frac{z_{0}^{*}\left[\left(z_{0}-z_{0}^{*}\right) \bar{M}+\bar{M}_{0}\right]}{\bar{z}_{0}\left(z-z_{0}^{*}\right)^{2}}-\frac{R^{2} \bar{\Gamma}^{\prime}}{z^{2}}+\Omega_{0}(z),
$$

where

$$
z_{0}^{*}=R^{2} / \bar{z}_{0}
$$

$\Omega_{0}(z)$ is also a holomorphic function in the entire plane cut along $L$.

Taking the derivative of (1) with respect to $\theta$ and noting that $z=t$ on $L$, we obtain

$$
\left(u^{\prime}+i v^{\prime}\right)^{+}=\left(u^{\prime}+i v^{\prime}\right)^{-}=i t H\left(\epsilon_{1}, \ldots, \epsilon_{n}\right) \text { on } L
$$

where

$$
H\left(\epsilon_{1}, \ldots, \epsilon_{n}\right)= \begin{cases}i \epsilon_{1} & \text { on } L_{1} \\ \cdots & \\ i \epsilon_{n} & \text { on } L_{n} .\end{cases}
$$

Substituting (16) into (19), we obtain

$$
\begin{array}{ll}
\kappa \Phi^{+}(t)-\Omega^{-}(t)=2 \mu H & \text { on } L \\
\kappa \Phi^{-}(t)-\Omega^{+}(t)=2 \mu H & \text { on } L .
\end{array}
$$

The addition and subtraction of (21) and (22) yield

$$
\begin{gathered}
{[\kappa \Phi(t)-\Omega(t)]^{+}+[\kappa \Phi(t)-\Omega(t)]^{-}=4 u H \text { on } L} \\
{[\kappa \Phi(t)+\Omega(t)]^{+}-[\kappa \Phi(t)+\Omega(t)]^{-}=0 \text { on } L .}
\end{gathered}
$$

Substituting (10) and (17) into (24), we obtain

$$
\left[\kappa \Phi_{0}(t)+\Omega_{0}(t)\right]^{+}-\left[\kappa \Phi_{0}(t)+\Omega_{0}(t)\right]^{-}=0 \text { on } L .
$$

According to Liouville's theorem, $\kappa \Phi_{0}(z)+\Omega_{0}(z)$ is a constant in the entire plane:

$$
\kappa \Phi_{0}(z)+\Omega_{0}(z)=D .
$$

Substituting (10) and (17) into (23) and using (26), we obtain

$$
\begin{gathered}
\Phi_{0}^{+}(t)+\Phi_{0}^{-}(t)=f(t) \text { on } L, \\
f(t)=-\frac{M}{t-z_{0}}-\frac{\bar{N}}{\kappa t}+\frac{\bar{N}}{\kappa\left(t-z_{0}^{*}\right)}-\frac{z_{0}^{*}\left[\left(z_{0}-z_{0}^{*}\right) \bar{M}+\bar{M}_{0}\right]}{\kappa \bar{z}_{0}\left(t-z_{0}^{*}\right)^{2}}-\frac{R^{2} \bar{\Gamma}^{\prime}}{\kappa t^{2}}-\Gamma+\frac{D}{\kappa}+\frac{2 \mu H}{\kappa} .
\end{gathered}
$$

The general solution of eq. (27) is

$$
\Phi_{0}(z)=\frac{x_{o}(z)}{2 \pi i} \int_{L} \frac{f(t)}{x_{0}^{+}(t)} \cdot \frac{\mathrm{d} t}{t-z}+x_{o}(z) p(z),
$$

where

$$
x_{0}(z)=\prod_{j=1}^{n}\left(z-a_{j}\right)^{-1 / 2}\left(z-b_{j}\right)^{-1 / 2}
$$

which is a single-valued branch in the plane cut along $L$ and for which

$$
\lim _{|z| \rightarrow \infty} z^{n} x_{0}(z)=1
$$


$P(z)$ is an arbitrary polynomial consistent with the behavior of $\Phi_{0}(z)$ at infinity:

$$
P(z)=C_{1} z^{n-1}+C_{2} z^{n-2}+\cdots+C_{n} .
$$

In the general solution given by (29), complex constants $D, C_{1}, \ldots, C_{n}$ and real constants $\epsilon_{1}, \ldots, \epsilon_{n}$ will be determined from equilibrium conditions (4) and (5), as well as from the behavior of $\Psi(z)$ at $z=0$. From (14), it is seen that

$$
\Psi(z)=\frac{R^{2}}{z^{2}} \Phi(z)-\frac{R^{2}}{z^{2}} \Omega\left(\frac{R^{2}}{z}\right)-\frac{R^{2}}{z} \Phi^{\prime}(z) .
$$

The condition that $\Psi(z)$ is holomorphic at $z=0$ leads to

$$
\bar{D}=\Phi(0) \text {. }
$$

Substituting (15) into (4), we obtain

$$
\int_{a_{j}}^{b_{j}}\left[\Phi^{+}(t)+\Omega^{-}(t)\right] \mathrm{d} t-\int_{a_{j}}^{b_{j}}\left[\Phi^{-}(t)+\Omega^{+}(t)\right] \mathrm{d} t=0 \quad j=1, \ldots, n,
$$

which can be reduced to integrals along closed contours:

$$
\oint_{A_{j}}[\Phi(z)-\Omega(z)] \mathrm{d} z=0 \quad j=1, \ldots, n,
$$

where $A_{j}$ are clockwise closed contours encircling rigid arcs $L_{j}$ with poles $\left(z=z_{0}, z_{0}^{*}, 0\right)$ outside.

Similarly, the substitution of (15) into (5) yields

$$
R_{e} \oint_{A_{j}} \frac{1}{z}[\Phi(z)-\Omega(z)] \mathrm{d} z=0 \quad j=1, \ldots, n .
$$

The set of $(2 n+1)$ linear algebraic equations given by (34), (36) and (37) determines the remaining constants $D, C_{1}, \ldots, C_{n}$ and $\epsilon_{1}, \ldots, \epsilon_{n}$.

On the other hand, for large values of $|z|$, we have

$$
\Phi(z)=\Gamma+\frac{M}{z}+O\left(\frac{1}{z^{2}}\right)
$$

From (10), (29) and (38), it is seen that

$$
C_{1}=0 \text {. }
$$

It can be shown that (39) may replace one of eqs (36) if rigid arcs are traction-free.

Finally, we point out that if tractions, which have resultant vectors $P_{j}^{*}+i Q_{j}^{*}$ and resultant moments about the origin, $M_{j}^{*}$, are applied on rigid arcs $L_{j}$, the following equations

$$
\begin{gathered}
\oint_{\Lambda_{j}}[\Phi(z)-\Omega(z)] \mathrm{d} z=-Q_{j}^{*}+i P_{j}^{*} \quad j=1, \ldots, n \\
R_{\mathrm{e}} \oint_{\Lambda_{j}} \frac{1}{z}[\Phi(z)-\Omega(z)] \mathrm{d} z=-\frac{M_{j}^{*}}{R^{2}} \quad j=1, \ldots, n
\end{gathered}
$$

will take the place of (36) and (37).

\section{EXAMPLES}

In the following, we consider the application of the general solution given by (29) to the case of a rigid arc, which may have some practical importance (Fig. 4). For this case, (28) and (30) become

$$
\begin{gathered}
f(t)=-\frac{M}{t-z_{0}}-\frac{N}{\kappa t}+\frac{\bar{N}}{\kappa\left(t-z_{0}^{*}\right)}-\frac{z_{0}^{*}\left[\left(z_{0}-z_{0}^{*}\right) \bar{M}+\bar{M}_{0}\right]}{\kappa \bar{z}_{0}\left(t-z_{0}^{*}\right)^{2}}-\frac{R^{2} \Gamma^{\prime}}{\kappa t^{2}}-\Gamma+\frac{D}{\kappa}+\frac{2 \mu \epsilon_{i}}{\kappa} \\
x_{0}(z)=(z-a)^{-1 / 2}(z-b)^{-1 / 2}=\left(z^{2}-2 R z \cos \theta+R^{2}\right)^{-1 / 2} .
\end{gathered}
$$




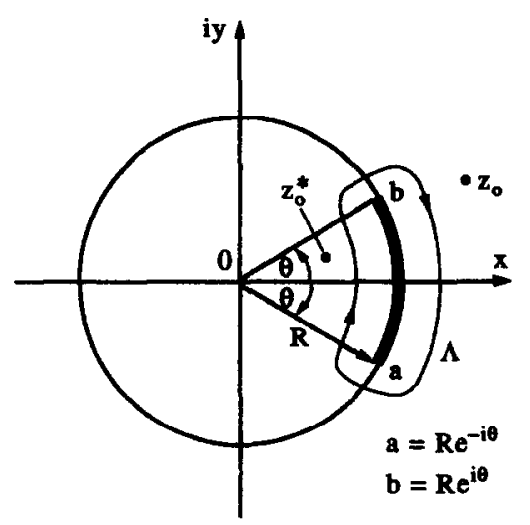

Fig. 4. Integral round a contour.

To integrate (29), we consider the following integral:

$$
I(z)=\frac{1}{2 \pi i} \oint_{\Lambda} \frac{f(\xi)}{x_{0}(\xi)} \cdot \frac{\mathrm{d} \xi}{\xi-z},
$$

where $A$ is a clockwise closed contour encircling the rigid arc with poles $\left(z=0, z_{0}, z_{0}^{*}\right)$ outside, as shown in Fig. 4. According to Cauchy's formula for the infinite region, we have

$$
I(z)=f(z) / x_{0}(z)-G(z)
$$

where $G(z)$ is the sum of the principal parts of $f(z) / x_{0}(z)$ at the poles and at infinity. It should be pointed out that the constant term of the expansion of a function in the vicinity of $z=\infty$ is included in the principal part. Noting that

$$
\begin{aligned}
& x_{0}^{+}(t)=-x_{0}^{-}(t) \text { on } L \\
& x_{0}^{+}(t)=x_{0}^{-}(t) \text { on } L^{\prime},
\end{aligned}
$$

we see that the integral in (29) can be achieved by shrinking the contour $\Lambda$ to the rigid arc:

$$
\frac{1}{2 \pi i} \int_{L} \frac{f(t)}{x_{0}^{+}(t)} \cdot \frac{\mathrm{d} t}{t-z}=\frac{1}{2}\left[\frac{1}{2 \pi i} \int_{L} \frac{f(t)}{x_{0}^{+}(t)} \cdot \frac{\mathrm{d} t}{t-z}-\frac{1}{2 \pi i} \int_{L} \frac{f(t)}{x_{0}^{-}(t)} \cdot \frac{\mathrm{d} t}{t-z}\right]=\frac{1}{2} I(z) .
$$

Finally, from (10), (17), (26), (30), (39), (45) and (48), we obtain

$$
\begin{gathered}
\Phi(z)=\frac{M}{z-z_{0}}+\Gamma+\frac{1}{2} f(z)-\frac{1}{2} x_{0}(z) G(z) \\
\Omega(z)=-\frac{\bar{N}}{z}+\frac{\bar{N}}{z-z_{0}^{*}}-\frac{z_{0}^{*}\left[\left(z_{0}-z_{0}^{*}\right) \bar{M}+\bar{M}_{0}\right]}{\bar{z}_{0}\left(z-z_{0}^{*}\right)^{2}}-\frac{R^{2} \bar{\Gamma}^{\prime}}{z^{2}}+D-\frac{\kappa}{2} f(z)+\frac{\kappa}{2} x_{0}(z) G(z) .
\end{gathered}
$$

Example 1. An arbitrary uniform stress state at infinity

For this case, (42) becomes

$$
f(t)=-\frac{R^{2} \bar{\Gamma}^{\prime}}{\kappa t^{2}}-\Gamma+\frac{D}{\kappa}+\frac{2 \mu \epsilon i}{\kappa} .
$$

Noting that $1 / x_{0}(z)$ has the expansions

$$
1 / x_{0}(z)=-R+z \cos \theta-\frac{\sin ^{2} \theta}{2 R} z^{2}+\cdots
$$


in the vicinity of $z=0$, and

$$
1 / x_{0}(z)=z-R \cos \theta+\frac{R^{2} \sin ^{2} \theta}{2 z}+\cdots
$$

in the vicinity of $z=\infty, G(z)$, the sum of the principal parts of $f(z) / x_{0}(z)$ at $z=0, \infty$ is easy to separate:

$$
G(z)=\frac{R^{3} \bar{\Gamma}^{\prime}}{\kappa z^{2}}-\frac{R^{2} \bar{\Gamma}^{\prime} \cos \theta}{\kappa z}+\left(-\Gamma+\frac{D}{\kappa}+\frac{2 \mu \epsilon i}{\kappa}\right)(z-R \cos \theta) .
$$

Hence the general solution given by (49) and (50) becomes

$$
\begin{aligned}
& \Phi(z)=\frac{1}{2}\left(-\frac{R^{2} \bar{\Gamma}^{\prime}}{\kappa z^{2}}+\Gamma+\frac{D}{\kappa}+\frac{2 \mu \epsilon i}{\kappa}\right)+\frac{x_{0}(z)}{2}\left(-\frac{R^{3} \bar{\Gamma}^{\prime}}{\kappa z^{2}}+\frac{R^{2} \bar{\Gamma}^{\prime} \cos \theta}{\kappa z}\right) \\
& +\frac{x_{0}(z)}{2}\left(\Gamma-\frac{D}{\kappa}-\frac{2 \mu \epsilon i}{\kappa}\right)(z-R \cos \theta) \\
& \mathbf{\Omega}(z)=\frac{1}{2}\left(-\frac{R^{2} \Gamma^{\prime}}{z^{2}}+\kappa \Gamma+D-2 \mu \epsilon i\right)-\frac{x_{0}(z)}{2}\left(-\frac{R^{3} \Gamma^{\prime}}{z^{2}}+\frac{R^{2} \bar{\Gamma}^{\prime} \cos \theta}{z}\right) \\
& -\frac{x_{0}(z)}{2}(\kappa \Gamma-D-2 \mu \epsilon i)(z-R \cos \theta) .
\end{aligned}
$$

Substituting (55) and (56) into (37) and using the residue theorem, we obtain

$$
\epsilon=-\frac{\left(\Gamma^{\prime}-\bar{\Gamma}^{\prime}\right) \cos ^{2} \frac{\theta}{2}}{4 \mu i}-\frac{D-\bar{D}}{4 \mu i}
$$

From (34) it is seen that

$$
\begin{gathered}
I_{m} D=0 \\
D=\left[\Gamma \cos ^{2} \frac{\theta}{2}-\frac{\left(\Gamma^{\prime}+\bar{\Gamma}^{\prime}\right) \sin ^{2} \theta}{8 \kappa}\right] /\left(1-\frac{\sin ^{2} \frac{\theta}{2}}{\kappa}\right) .
\end{gathered}
$$

Equation (57) becomes

$$
\epsilon=-\frac{\left(\Gamma^{\prime}-\bar{\Gamma}^{\prime}\right) \cos ^{2} \frac{\theta}{2}}{4 \mu i}
$$

For the case of uniaxial tension at infinity $\left(\sigma_{1}=\sigma, \sigma_{2}=0\right)$,

$$
\Gamma=\frac{\sigma}{4} \quad \bar{\Gamma}^{\prime}=-\frac{1}{2} \sigma \mathrm{e}^{-2 i x},
$$

so that

$$
\epsilon=-\frac{\sigma \sin 2 \alpha \cos ^{2} \frac{\theta}{2}}{4 \mu}
$$

Example 2. The resultant moment of tractions which are applied on the rigid arc is $\mathrm{M}^{*}$ (the resultant vector vanishes)

Noting that (39) still holds for this case, (42), (49) and (50) give

$$
f(t)=\frac{D}{\kappa}+\frac{2 \mu \epsilon i}{\kappa}
$$




$$
\begin{aligned}
& \Phi(z)=\frac{D}{2 \kappa}+\frac{\mu \epsilon i}{\kappa}-\frac{x_{0}(z)}{2}\left(\frac{D}{\kappa}+\frac{2 \mu \epsilon i}{\kappa}\right)(z-R \cos \theta) \\
& \Omega(z)=\frac{D}{2}-\mu \epsilon i+\frac{x_{0}(z)}{2}(D+2 \mu \epsilon i)(z-R \cos \theta) .
\end{aligned}
$$

The substitution of (64) and (65) into (34) and (41) yields

$$
\begin{gathered}
D=-\frac{M^{*} i}{2 \pi R^{2}(1+\kappa)} \\
\epsilon=\frac{M^{*}}{4 \pi \mu(1+\kappa) R^{2} \sin ^{2} \frac{\theta}{2}}\left(\kappa+\sin ^{2} \frac{\theta}{2}\right) .
\end{gathered}
$$

Example 3. A concentrated force $\mathrm{P}+\mathrm{iQ}$ and a moment $\mathrm{m}$ at an arbitrary point $\mathrm{z}_{0}$

In the same manner as in the above two examples, we obtain

$$
\begin{aligned}
f(t)= & -\frac{M}{t-z_{0}}-\frac{\bar{N}}{\kappa t}+\frac{\bar{N}}{\kappa\left(t-z_{0}^{*}\right)}-\frac{z_{0}^{*}\left[\left(z_{0}-z_{0}^{*}\right) \bar{M}+\bar{M}_{0}\right]}{\kappa \bar{z}_{0}\left(t-z_{0}^{*}\right)^{2}}+\frac{D}{\kappa}+\frac{2 \mu \epsilon i}{\kappa} \\
\Phi(z)= & \frac{1}{2}\left\{\frac{M}{z-z_{0}}-\frac{\bar{N}}{\kappa z}+\frac{\bar{N}}{\kappa\left(z-z_{0}^{*}\right)}-\frac{z_{0}^{*}\left[\left(z_{0}-z_{0}^{*}\right) \bar{M}+\bar{M}_{0}\right]}{\kappa \bar{z}_{0}\left(z-z_{0}^{*}\right)^{2}}+\frac{D}{\kappa}+\frac{2 \mu \epsilon i}{\kappa}\right\} \\
& -\frac{x_{0}(z)}{2}\left[-\frac{1}{x_{0}\left(z_{0}\right)} \cdot \frac{M}{z-z_{0}}-M+\frac{R \bar{N}}{\kappa z}+\frac{1}{x_{0}\left(z_{0}^{*}\right)} \cdot \frac{\bar{N}}{\kappa\left(z-z_{0}^{*}\right)}\right] \\
& +\frac{x_{0}(z)}{2 x_{0}\left(z_{0}^{*}\right)} \frac{z_{0}^{*}\left[\left(z_{0}-z_{0}^{*}\right) \bar{M}+\bar{M}_{0}\right]}{\kappa \bar{z}_{0}} \cdot\left[\frac{1}{\left(z-z_{0}^{*}\right)^{2}}+\frac{1}{z-z_{0}^{*}} \cdot \frac{2 z_{0}^{*}-a-b}{2\left(z_{0}^{*}-a\right)\left(z_{0}^{*}-b\right)}\right] \\
& -\frac{1}{2} x_{0}(z)\left(\frac{D}{\kappa}+\frac{2 \mu \epsilon i}{\kappa}\right)\left(z-\frac{1}{2} a-\frac{1}{2} b\right) \\
\Omega(z)= & \frac{1}{2}\left\{\frac{\kappa M}{z-z_{0}}-\frac{\bar{N}}{z}+\frac{\bar{N}}{z-z_{0}^{*}}-\frac{z_{0}^{*}\left[\left(z_{0}-z_{0}^{*}\right) \bar{M}+\bar{M}_{0}\right]}{\bar{z}_{0}\left(z-z_{0}^{*}\right)^{2}}+D-2 \mu \epsilon i\right\} \\
+ & \frac{x_{0}(z)}{2}\left[-\frac{1}{x_{0}(z)} \cdot \frac{\kappa M}{z-z_{0}}-\kappa M+\frac{R \bar{N}}{z}+\frac{1}{x_{0}\left(z_{0}^{*}\right)} \cdot \frac{\bar{N}}{z-z_{0}^{*}}\right] \\
+ & \frac{x_{0}(z)}{2 x_{0}\left(z_{0}^{*}\right)} \cdot \frac{z_{0}^{*}\left[\left(z_{0}-z_{0}^{*}\right) \bar{M}+\bar{M}_{0}\right]}{\bar{z}_{0}}\left[\frac{1}{\left(z-z_{0}^{*}\right)^{2}}+\frac{1}{z-z_{0}^{*}} \cdot \frac{2 z_{0}^{*}-a-b}{2\left(z_{0}^{*}-a\right)\left(z_{0}^{*}-b\right)}\right] \\
+ & \frac{1}{2} x_{0}(z)(D+2 \mu \epsilon i)\left(z-\frac{1}{2} a-\frac{1}{2} b\right) \cdot \\
+ & \\
& \\
&
\end{aligned}
$$

Similarly, $D$ and $\epsilon$ can be determined from (34) and (37).

\section{STRESS DISTRIBUTION AT THE RIGID CIRCULAR ARC END}

It is of interest to examine the stress distribution in the immediate vicinity of the rigid circular arc end to show the effect of curvature. It is shown that the singular behavior of the stresses remains proportional to the inverse square root of $\rho$ (i.e. $\rho^{-1 / 2}$ ), as in the case of the immediate vicinity of the arc crack tip, where $\rho$ is the distance from the rigid arc end. As an example, we discuss the case of a rigid circular arc subjected to a uniaxial tension at infinity $\left(\sigma_{1}=\sigma, \sigma_{2}=0\right)$. Introducing 


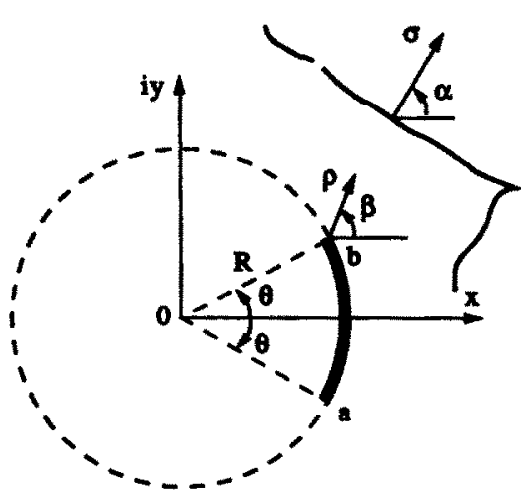

Fig. 5. Polar coordinate near a rigid circular arc end.

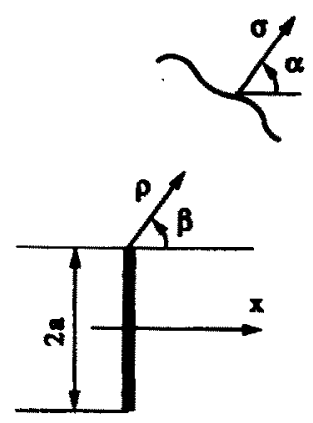

Fig. 6. Polar coordinate near a rigid line end.

the polar coordinates $(\rho, \beta)$ at the rigid arc end $b$ (Fig. 5), from eqs (6), (15), (33), (55) and (56), and noting (59)-(62), we obtain

$$
\begin{aligned}
& \left.\sigma_{F}=\frac{\sigma}{8 \sqrt{ } 2} \sqrt{\left(\frac{R}{\sin \theta}\right.} \frac{2}{\rho}\right)\left\{\frac { 2 } { \kappa } \left[\cos \left(45^{\circ}-\theta-\frac{\beta}{2}\right)+\cos (\theta-\beta) \cos \left(45^{\circ}-\frac{3}{2} \beta\right)-2 \cos \left(45^{\circ}+\theta-\frac{\beta}{2}\right)\right.\right. \\
& \left.+\left[1-\frac{\cos ^{2} \theta-\frac{3}{8 \kappa} \sin ^{2} \theta}{\kappa-\sin ^{2} \frac{\theta}{2}}\right]\left[(1-\kappa) \cos \left(45^{\circ}-\frac{\beta}{2}\right)-\cos (\theta-\beta) \cdot \cos \left(45^{\circ}+\theta-\frac{3}{2} \beta\right)\right]\right\}
\end{aligned}
$$

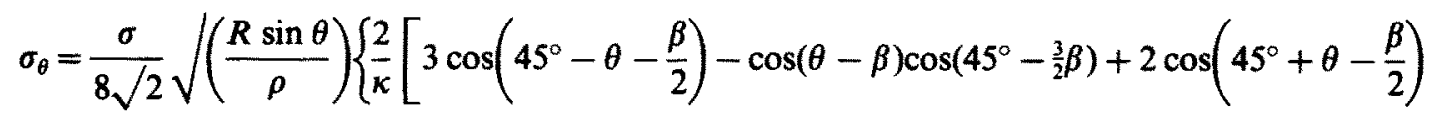

$$
\begin{aligned}
& \left.+\left\{1-\frac{\cos ^{2} \theta-\frac{3}{8 \kappa} \sin ^{2} \theta}{\kappa-\sin ^{2} \frac{\theta}{2}}\right]\left[(3+\kappa) \cdot \cos \left(45^{\circ}-\frac{\beta}{2}\right)-\cos (\theta-\beta) \cdot \cos \left(45^{\circ}+\theta-\frac{3}{2} \beta\right)\right]\right\} \\
& \tau_{r \theta}=\frac{\sigma}{8 \sqrt{ } 2} \sqrt{\left(\frac{R \sin \theta}{\rho}\right)}\left\{\frac{2}{\kappa}\left[\cos \left(45^{\circ}+\theta+\frac{\beta}{2}\right)-\cos (\theta-\beta) \cos \left(45^{\circ}+\frac{3}{2} \beta\right)\right]+2 \cos \left(45^{\circ}-2 \theta+\frac{\beta}{2}\right)\right. \\
& +\left[1-\frac{\cos ^{2} \theta-\frac{3}{8 \kappa} \sin ^{2} \theta}{\kappa-\sin ^{2} \frac{\theta}{2}}\right] \cdot\left[\cos \left(45^{\circ}+\frac{\beta}{2}\right)+\kappa \cos \left(45^{\circ}-\theta+\frac{\beta}{2}\right)\right. \\
& \left.\left.-\cos (\theta-\beta) \cos \left(45^{\circ}-\theta+\frac{3}{2} \beta\right)\right]\right\} \text { for } \alpha=90^{\circ}, \rho \ll R \text {. }
\end{aligned}
$$

Letting $R \rightarrow \infty, \theta \rightarrow 0$, but keeping $R \sin \theta=a$, we arrive at the case as shown in Fig. 6 , and as $\sigma_{r} \rightarrow \sigma_{x}, \sigma_{\theta} \rightarrow \sigma_{y}, \tau_{r \theta} \rightarrow \tau_{x y}$, by (71) we have

$$
\begin{aligned}
\sigma_{x} & =\frac{\sigma}{8 \sqrt{2}} \sqrt{\left(\frac{a}{\rho}\right)}\left[\left(\frac{1}{\kappa}-\kappa\right) \sin \left(45^{\circ}+\frac{\beta}{2}\right)+\left(1+\frac{1}{\kappa}\right) \cos \beta \sin \left(45^{\circ}+\frac{3}{2} \beta\right)\right] \\
\sigma_{y} & =\frac{\sigma}{8 \sqrt{2}} \sqrt{\left(\frac{a}{\rho}\right)}\left[\left(4+\kappa+\frac{3}{\kappa}\right) \sin \left(45^{\circ}+\frac{\beta}{2}\right)-\left(1+\frac{1}{\kappa}\right) \cos \beta \sin \left(45^{\circ}+\frac{3}{2} \beta\right)\right] \\
\tau_{x y} & =-\frac{\sigma}{8 \sqrt{ } 2} \sqrt{\left(\frac{a}{\rho}\right)}\left[\left(2+\kappa+\frac{1}{\kappa}\right) \sin \left(45^{\circ}-\frac{\beta}{2}\right)+\left(1+\frac{1}{\kappa}\right) \cos \beta \sin \left(45^{\circ}-\frac{3}{2} \beta\right)\right]
\end{aligned}
$$

for $\alpha=90^{\circ}, \rho \ll a$. 
Letting $\beta^{\prime}=90^{\circ}+\beta$, and noticing the difference of the convenients, from (72), we obtain results in agreement with ref. [5].

\section{REFERENCES}

[1] J. D. Eshelby, The determination of the elastic field of an ellipsoidal inclusion, and related problems. Proc. R. Soc. Lond. A241, 376-396 (1957).

[2] N. I. Muskhelishvili, Some Basic Problems of Mathematical Theory of Elasticity. Noordhoff, Leyden (1975).

[3] T. H. Hao and Y. W. Wu, Elastic plane problem of collinear periodical rigid lines. Engng Fracture Mech. 33, 979-981 (1989).

[4] C. P. Jiang, The plane problem of collinear rigid lines under arbitrary loads. Engng Fracture Mech. 39, $299-308$ (1991).

[5] X. Markenscoff and J. Dundurs, Weight functions for rigid line inclusions. Proc. 7th Int. Conf. on Fracture, Houston, TX, pp. 37-39 (March 1989)

[6] Y. W. Liu and C. P. Jiang, Problem on circular arc cracks between bonded dissimilar under concentrated force and moment. Appl. Math. Mech. 8(3), 271 (1987).

(Received 27 January 1993) 